\title{
GENERALIZED THEODORSEN SOLUTION FOR SINGULAR INTEGRAL EQUATIONS OF THE AIRFOIL CLASS*
}

BY

\author{
M. H. WILLIAMS
}

Princeton University

1. Introduction. A class of singular integral equations is considered which arise in various two-dimensional mixed-boundary-value problems with simple harmonic time variation. The prototypical problem in this class is the determination of the lifting pressure distribution on an oscillating airfoil in an unbounded incompressible potential flow. The solution of this problem was first determined by Theodorsen [1] and independently by Kussner [2] and others. Their results are discussed in many modern books on aerodynamics and aeroelasticity (cf. [3]). In the present paper it will be shown that Theodorsen's solution, with some modification, is valid for a general class of unsteady kernel functions, of which that considered by Theodorsen et al. is but one member.

The singular integral equation in question is the generalized airfoil equation:

$$
f_{0}^{1} d t K(t-x ; k) \phi(t ; k)=w(x ; k)
$$

for the function $\phi(x ; k)$ which is presumed to be integrable over the interval $[0,1]$. The kernel function $K(x ; k)$ is assumed to be regular everywhere except at $x=0$ where it has a strong first-order singularity (the integral in (1) being a Cauchy principal value). The problem, then, is to determine the function (or set of functions) $\phi$ which satisfies (1) for a given $w(x, k)$ in $(0,1)$.

The parameter $k$, which appears implicitly in (1), represents the frequency (suitably nondimensionalized) of the simple harmonic motion associated with the forcing function $w$. The class of problems to be addressed here is defined by the following specification of the frequency dependence of the kernel $K$ :

$$
K(x ; k)=K_{0}(x)-i k \exp (i k x) \int_{x}^{\infty} d t \exp (-i k t) K_{0}(t)
$$

where $K_{0}(x)=K(x ; 0)$ is the corresponding steady kernel function. In an unbounded incompressible potential flow:

$$
K_{0}(x)=-1 / \pi x .
$$

Theodorsen solved (1) for the particular kernel defined by (2) and (3). We shall solve (1) for kernels of type (2) but with a more general steady kernel $K_{0}(x)$ which need only be consistent with the presumed properties of $K$.

\footnotetext{
* Received August 25, 1976; revised version received November 22, 1976. The author expresses his gratitude to Professor E. H. Dowell for his aid in the preparation of this paper. This work is supported by NASA, Ames Research Center under Grant NGR-31-001-197.
} 
It should be noted that in practical applications (which will be discussed more fully in Sec. (8)) the kernel $K(x ; k)$ (and so, also $\left.K_{0}(x)\right)$ vanishes as $x \rightarrow+\infty$. This must be so if (by Eq. (1) $w(x, k)$ is to vanish as $x \rightarrow-\infty$, which, in aerodynamics, corresponds to points far upstream from the airfoil. Conversely, disturbances need not and in general do not decay downstream. The kernel (2) is consistent with such an asymmetry of the far field disturbances.

The general solution of (1) can be written in the "resolvent" form:

$$
\phi(x ; k)=\alpha h(x ; k)+f_{0}^{1} d t \Gamma(t, x ; k) w(t ; k)
$$

where $h(x ; k)$ is the (integrable) homogeneous solution of $(1), \alpha$ is an arbitrary constant, and $\Gamma(t, x ; k)$ is the "resolvent" function of the kernel $K$. For the sake of definiteness these functions can be made to satisfy the conditions:

$$
\begin{array}{r}
\int_{0}^{1} h(x ; k) d x=1, \\
\Gamma(t, 1 ; k)=0 .
\end{array}
$$

In [4] it was shown that the resolvent of any singular integral equation of type (1) can be written explicitly in terms of the homogeneous solution, $h(x ; k)$, and a particular solution, $\phi_{1}(x, k)$, say, which satisfies $(1)$ for $w=1$ in $(0,1)$ and $\phi_{1}(1 ; k)=0$. Thus in general two frequency-dependent functions $h$ and $\phi_{1}$, are required to construct the solution of (1).

Using the theory developed in [4] we shall show that for kernel functions of the form (2) a complete separation of the spatial and frequency dependence of the solution is possible. Specificially we shall show that the unsteady resolvent $\Gamma(t, x ; k)$ and the unsteady homogeneous solution $h(x ; k)$ can be written directly in terms of the steady solutions $\phi_{1}(x$; $0)$ and $h(x ; 0)$ and a single frequency-dependent function $C(k)$ which reduces to the wellknown "Theodorsen function" for the steady kernel (3).

2. An equivalent steady problem. We wish to write Eq. (1) in the form:

$$
\int_{0}^{1} K_{0}(t-x) \phi(t ; k) d t=\Omega(x ; k) .
$$

To do this we solve (2) for $K_{0}$ :

$$
K_{0}(x)=-\exp (i k x) \frac{d}{d x} \exp (-i k x) \int_{x}^{\infty} d t K(t ; k) .
$$

Thus, by substituting (8) into (7) and using the fact that $\phi(t ; k)$ is a solution of (1) we find that:

$$
\Omega(x ; k)=w(x ; k)+i k \int_{-\infty}^{x} d t w(t ; k)
$$

The problem posed by (7) and (9) is fully equivalent to the original problem (1). If $\Omega(x$; $k$ ) were known in $[0,1]$ then $(7)$ would constitute an essentially steady problem with $k$ carried as an irrelevant parameter. However, since $w$ is specified on $[0,1]$ only, it is clear from (9) that $\Omega$ is a functional of the solution, depending on the behavior of $w(x ; k)$ for $x$ $<0$. For values of $x$ in $[0,1], \Omega$ may be split into a variable known part and a constant unknown part: 


$$
\Omega(x ; k)=w(x ; k)+i k A(k), x \in(0,1)
$$

where

$$
\bar{w}(x ; k)=w(x ; k)+i k \int_{0}^{x} d t w(t ; k)
$$

is known for $x$ in $(0,1)$, and:

$$
A(k)=\int_{-\infty}^{0} d x w(x ; k)
$$

is an as-yet-undetermined constant.

Comparing (7) and (10) we see that the solution may also be split:

$$
\phi(x ; k)=\bar{\phi}(x ; k)+i k A \phi_{01}(x)
$$

where:

$$
\int_{0}^{1} d t K_{0}(t-x) \bar{\phi}(t ; k)=\bar{w}(x ; k)
$$

and

$$
\int_{0}^{1} d t K_{0}(t-x) \phi_{01}(t)=w_{01}(x)=1 \text { for } x \in(0, \dot{1})
$$

Thus the original problem (1) has been reduced to the two steady problems $(14,15)$ and the question of determing the constant $A$. We shall return to the former task in Sec. (4) after addressing the latter question in the next section.

3. Determination of $\mathbf{A}(\mathbf{k})$ If we multiply (9) by $\exp (i k x)$ and integrate over $(-\infty, 0)$ we find that:

$$
A(k) \equiv \int_{-\infty}^{0} d x w(x, k)=\int_{-\infty}^{0} d x \exp (i k x) \Omega(x ; k)
$$

But, from (7) and (13-15), it is apparent that:

$$
\Omega(x ; k)=\bar{w}(x ; k)+i k A w_{01}(x)
$$

which is valid for all $x$ in $(-\infty, \infty)$ (note that (17) reduces to (10) for $x$ in $[0,1])$. Thus, combining (17) and (16) and solving for $A$, we obtain:

$$
A(k)=\int_{-\infty}^{0} d x \exp (i k x) \bar{w}(x ; k) /\left(1-i k c_{01}(k)\right)
$$

where we have defined:

$$
c_{01}(k)=\int_{-\infty}^{0} d x \exp (i k x) w_{01}(x)
$$

Eqs. (18) and (19) relate $A$ directly to the solution of the steady problems (14) and (15), which we must now consider in greater detail.

4. The solution of the steady problem. In this section we review the principal results of [4] on the solution of singular integral equations of the type (14) (or (1)). In that paper it was shown that the general solution could be constructed from two solutions $\phi_{00}$ and $\phi_{01}$ 
which are defined by:

$$
\int_{0}^{1} d t K_{0}(t-x) \phi_{0 n}(t)=w_{0 n}(x)
$$

where:

$$
\begin{array}{ll}
w_{00}(x)=0, x \in[0,1] ; & \int_{0}^{1} d x \phi_{00}(x)=1, \\
w_{01}(x)=1, x \in[0,1] ; & \phi_{01}(1)=0 .
\end{array}
$$

Then if $g(x)$ is a given function on $(0,1)$ and

$$
\int_{0}^{1} d t K_{0}(t-x) \psi(t)=g(x)
$$

then the general solution for $\psi$ is:

$$
\psi(x)=\alpha \phi_{00}(x)+\int_{0}^{1} d t g(t) \Gamma_{0}(t, x)
$$

where $\alpha$ is an arbitrary constant,

$$
\begin{aligned}
\Gamma_{0}(t, x) & \equiv \phi_{01}(x) \phi_{00}(1-t)-\frac{\partial}{\partial t} G_{0}(t, x) \\
& =\phi_{01}(1-t) \phi_{00}(x)+\frac{\partial}{\partial x} G_{0}(t, x), \\
G_{0}(t, x) & \equiv-\int_{0}^{t} d s R_{0}(s, 1-x+t-s), \\
R_{0}(s, t) & =\phi_{01}(1-s) \phi_{00}(1-t)-\phi_{01}(1-t) \phi_{00}(1-s) .
\end{aligned}
$$

Upon substitution of (24) back into (23) we find that:

$$
g(x)=\alpha w_{00}(x)+\int_{0}^{1} d t g(t) w_{\Gamma_{0}}(t, x)
$$

where $w_{00}$ is defined by $(20,21)$ and

$$
w_{\Gamma_{0}}(t, x) \equiv \int_{0}^{1} d s K_{0}(s-x) \Gamma_{0}(t, s) .
$$

Eq. (28) is valid for all values of $x$.

A final useful result comes from combining (25a, b) with (29):

$$
\begin{array}{r}
w_{\Gamma_{0}}(t, x)=\phi_{00}(1-t) w_{01}(x)-\frac{\partial}{\partial t} w_{G_{0}}(t, x) \\
\phi_{01}(1-t) w_{00}(x)+\frac{\partial}{\partial x} w_{G_{0}}(t, x)
\end{array}
$$

where

$$
w_{G_{0}}(t, x)=\int_{0}^{1} d s K_{0}(s-x) G_{0}(t, s)
$$


Relation (30-31) was not stated explicitly in [4] in a form valid for all $x$. It was shown, however, that for values of $x$ in $[0,1]$ :

$$
\begin{aligned}
w_{G_{0}}(t, x) & =\int_{0}^{t} \phi_{00}(1-s) d s, \quad t<x<1 \\
& =-\int_{t}^{1} \phi_{00}(1-s) d s, \quad t>x>0,
\end{aligned}
$$

which is a unit step function in $x$. Thus, from $(30 \mathrm{a}, \mathrm{b})$ and $(32)$ :

$$
w_{\Gamma_{0}}(t, x)=\delta(t-x), x \in[0,1]
$$

(where $\delta$ is a Dirac delta function). This implies that (28) is an identity for $x \epsilon(0,1)$ as, of course, it must be.

We note the following special values of the functions defined above:

$$
\begin{aligned}
& \Gamma_{0}(t, 1)=0 \\
& G_{0}(t, 1)=G_{0}(t, 0)=G_{0}(1, x)=G_{0}(0, x)=0 .
\end{aligned}
$$

An important consequence of (35) is that the function $w_{G}(t, x)$, defined by (31), is continuous in $x$ across the end points of the interval. Specifically, by (32):

$$
\begin{aligned}
& w_{G_{0}}\left(t, 0^{-}\right)=w_{G_{0}}\left(t, 0^{+}\right)=-\int_{t}^{1} d s \phi_{00}(1-s), \\
& w_{G_{0}}\left(t, 1^{+}\right)=w_{G_{0}}\left(t, 1^{-}\right)=\int_{0}^{t} d s \phi_{00}(1-s) .
\end{aligned}
$$

It should, perhaps, be emphasized that all of the forgoing results are equally true of Eq. (1) as of Eq. (14). The simplification resulting from the assumption of the relation (2) between the steady and unsteady kernels rests entirely in our being able to separate the spatial and frequency dependencies of the solution.

It must be stated here that the foregoing results are based on the assumptions that the two functions $\phi_{00}(x)$ and $\phi_{01}(x)$ are known. In fact there appear to be no general means of finding these functions analytically. However, there are numerous well-known numerical methods for the approximate construction of such solutions. If such a method must be resorted to, then it will usually be simpler to construct the homogeneous solution, $\phi_{00}$, indirectly. To do this we define the "conjugate" of $\phi_{01}, \hat{\phi}_{01}$ say, by:

$$
\int_{0}^{1} K_{0}(t-x) \hat{\phi}_{01}(t) d t=\hat{w}_{01}(x)=1 \quad \text { for } \quad x \epsilon(0,1) ; \hat{\phi}_{01}(0)=0 .
$$

This conjugate solution satisfies the same equation as $\phi_{01}$ but with the side condition applied at the opposite boundary. Thus both $\phi_{01}$ and $\hat{\phi}_{01}$ may be evaluated by (practically) the same numerical scheme. The normalized homogeneous solution is, then, simply proportional to the difference:

$$
\phi_{00}(x)=\frac{1}{2 \bar{a}_{01}}\left[\phi_{01}(x)-\hat{\phi}_{01}(x)\right]
$$


where

$$
\dot{a_{01}} \equiv \frac{1}{2} \int_{0}^{1} d x\left[\phi_{01}(x)-\hat{\phi}_{01}(x)\right]
$$

In the very important special case where the kernel is antisymmetric, considerable simplification results, since then the solution of (38) is simply:

$$
\hat{\phi}_{01}(x)=-\phi_{01}(1-x) \text { if } K_{0}(-x)=-K_{0}(x)
$$

and thus

$$
\phi_{00}(x)=\frac{1}{2 a_{01}}\left[\phi_{01}(x)+\phi_{01}(1-x)\right]
$$

where

$$
a_{01} \equiv \int_{0}^{1} d x \phi_{01}(x) .
$$

For antisymmetric kernels, then, only one function, $\phi_{01}(x)$ need be evaluated numerically.

5. The unsteady resolvent. Given the results of the preceding section (as applied to the steady kernel) we are now able to write down the solution to the unsteady problem, i.e. to find the unsteady resolvent $\Gamma(t, x ; k)$ and unsteady homogeneous solution $h(x ; k)$ in terms of which that solution may be expressed (cf. (4)). In this section we shall concentrate on the resolvent. To this end we impose the restriction $\phi(1 ; k)=0$ on the solution (13) (i.e. $\alpha=0$ in (4)).

It follows from $(14)$ that, for $\bar{\phi}(1 ; k)=0$ :

$$
\bar{\phi}(x ; k)=\int_{0}^{1} d t \Gamma_{0}(t, x) \bar{w}(t ; k)
$$

where $\Gamma_{0}$ is the steady resolvent defined in (25).

Similarly, by (28), we can evaluate the variation of $w(x)$ for all $x$ :

$$
\bar{w}(x ; k)=\int_{0}^{1} d t \bar{w}(t, k) w_{\Gamma_{0}}(t, x) .
$$

Thus the constant $A(k)$ may be related to $w(x ; k)$ for $x$ in $[0,1]$ by combining (45) and (18):

$$
A(k)=\int_{0}^{1} d t \bar{w}(t ; k) c_{\Gamma}(t, k) /\left(1-i k c_{01}(k)\right)
$$

where $c_{01}$ is defined in (19) and

$$
c_{\Gamma}(t ; k) \equiv \int_{-\infty}^{0} d x \exp (i k x) w_{\Gamma_{0}}(t ; x) .
$$

Combining (44) and (46) in (13) we find that:

$$
\phi(x ; k)=\int_{0}^{1} d t \bar{w}(t ; k) \bar{\Gamma}(s, x ; k)
$$

where

$$
\Gamma(t, x ; k) \equiv \Gamma_{0}(t, x)+i k \phi_{01}(x) c_{\Gamma}(t ; k) /\left(1-i k c_{01}(k)\right)
$$


Recalling definition (11) of $\bar{w}$ in terms of $w$, we may write (48) directly in terms of $w$ (as in (4)), with the result that the unsteady resolvent is:

$$
\Gamma(t, x ; k)=\bar{\Gamma}(t, x ; k)+i k \int_{t}^{1} d s \Gamma(s, x ; k) .
$$

Using the form (25a) of $\Gamma_{0}(t, x)$ and the boundary values (35) of $G_{0}$ to compute the integral in (50), we may write this as:

$$
\Gamma(t, x ; k)=\Gamma_{0}(t, x)+i k G_{0}(t, x)+\phi_{01}(x) F(t ; k)
$$

where $G_{0}$ is defined in $(26)$ and

$$
F(t ; k)=\frac{i k}{1-i k c_{01}}\left[c_{\Gamma}(t ; k)+i k \int_{t}^{1} d s c_{\Gamma}(s ; k)\right]+i k \int_{t}^{1} d s \phi_{00}(1-s) .
$$

Now if (51) is compared to the corresponding solution found by Theodorsen for the special kernel (3), it is apparent that, at least in that case, the function $F(t, k)$ is simply proportional to $\phi_{01}(1-t)$. We shall now show that this is true for any steady kernel.

Using the two alternative forms $(30 \mathrm{a}, \mathrm{b})$ of $w_{\Gamma_{0}}$ to evaluate $c_{\Gamma}$ (from definition (47)) we find that:

$$
\begin{aligned}
c_{\Gamma}(t ; k) & =\phi_{00}(1-t) c_{01}(k)-\frac{\partial}{\partial t} c_{G}(t ; k) \\
& =\phi_{01}(1-t) c_{00}(k)+w_{G_{0}}\left(t, 0^{-}\right)-i k c_{G}(t ; k)
\end{aligned}
$$

where we have defined:

$$
\begin{gathered}
c_{00}(k)=\int_{-\infty}^{0} d x \exp (i k x) w_{00}(x), \\
c_{G}(t ; k)=\int_{-\infty}^{0} d x \exp (i k x) w_{G_{0}}(t ; x) .
\end{gathered}
$$

In obtaining (53b) an integration by parts was performed.

Now, from (53a):

$$
\int_{t}^{1} c_{\Gamma}(s, k) d s=c_{01}(k) \int_{t}^{1} d s \phi_{00}(1-s)+c_{G}(t ; k)
$$

where we have set $c_{G}(1, k)=0$ by (31) and (35). Thus, by combining (53b) and (56) we have:

$$
\begin{aligned}
c_{\Gamma}(t ; k)+i k \int_{t}^{1} d s c_{\Gamma}(s ; k) & =\phi_{01}(1-t) c_{00}(k)+w_{G_{0}}\left(t, 0^{-}\right) \\
& +i k c_{01} \int_{t}^{1} d s \phi_{00}(1-s)
\end{aligned}
$$

which, upon being inserted into (52), gives:

$$
F(t ; k)=\frac{i k c_{00}(k)}{1-i k c_{01}(k)} \phi_{01}(1-t)+\frac{i k}{1-i k c_{01}}\left[w_{G_{0}}\left(t, 0^{-}\right)+\int_{t}^{1} d s \phi_{00}(1-s)\right] .
$$

But by the continuity relation (36) the bracketed term in (58) vanishes, so that:

$$
F(t ; k)=\frac{i k c_{00}(k)}{1-i k c_{01}(k)} \phi_{01}(1-t) \text {. }
$$


We may thus write the unsteady resolvent in a form comparable to Theodorsen's solution:

$$
\Gamma(t, x ; k)=\Gamma_{0}(t, x)+i k G_{0}(t, x)-\frac{1}{\bar{a}_{01}}[1-C(k)] \phi_{01}(x) \phi_{01}(1-t)
$$

where

$$
C(k) \equiv 1+\frac{i k \bar{a}_{01} c_{00}(k)}{1-i k c_{01}(k)}
$$

and $\bar{a}_{01}$ is defined in (40).

The function $C(k)$, as will be seen below, plays the role of a generalized Theodorsen function.

6. The fundamental solutions. The resolvent (60) gives only a particular solution of the original integral equation (1). To complete the general solution (4) we must find the homogeneous solution $h$. This, however, is a simple task given the results of Secs. 2, 3 .

If $w(x, k) \equiv 0$ for all $x$ in $[0,1]$, then by $(11), \bar{w}(x, k)=0$ there, and thus, by $(13,14)$ :

$$
\begin{aligned}
& \phi(x ; k) \equiv h(x ; k)=\alpha \phi_{00}(x)+i k A \phi_{01}(x) \\
& \bar{w}(x, k)=\alpha w_{00}(x)
\end{aligned}
$$

where $\alpha$ is an arbitrary constant. The constant $A$, then, follows immediately from (18), (63) and (54):

$$
A=\alpha\left(c_{00} / 1-i k c_{01}\right)
$$

Imposing the normalization (5) to evaluate $\alpha$ and using definition (61), we find that:

$$
h(x ; k)=\left\{\phi_{00}(x)+\frac{1}{a_{01}}(C-1) \phi_{01}(x)\right\} /\left\{1+\frac{a_{01}}{\bar{a}_{01}}(C-1)\right\} .
$$

The results (65) and (60), then, give the general solution of Eq. (1). As remarked earlier, this solution could have been constructed, using the results of Sec. 4, from the homogeneous solution (60) and a particular solution of (1) for $w=1$ in $(0,1)$. These, then, are the "fundamental" solutions of the integral equations. Having already found the resolvent, however, it is a simple matter to compute the second fundamental solution:

$$
\begin{aligned}
\phi_{1}(x ; k) & \equiv \int_{0}^{1} d t \Gamma(t, x ; k) \\
& =\left[1-\frac{a_{01}}{\bar{a}_{01}}(1-C(k))\right] \phi_{01}(x)+i k \phi_{02}(x)
\end{aligned}
$$

where

$$
\phi_{02}(x) \equiv \int_{0}^{1} d t G_{0}(t, x)=\int_{x}^{1} d t\left[a_{01} \phi_{00}(t)-\phi_{01}(t)\right]
$$

The latter form of $\phi_{02}(x)$ follows most easily from (25b). It is of some interest to note that just as $\phi_{01}$ is the solution of the steady problem for constant $w$, so $\phi_{02}$ is the steady solution for linear $w$. Thus the unsteady solution for constant $w$, i.e. $\phi_{1}$, is equal to a steady solution for linear $w$. This may easily be generalized to say that the unsteady solution for 
any $n$ th-order polynomial $w$ will be equal to the steady solution for some $(n+1)$ th-order polynomial $w$ (this statement is clearly true in light of the equivalency of $(1)$ and $(7,8)$ ).

7. The generalized Theodorsen function. From Eqs. (38) and (39) we see that:

$$
w_{00}(x)=\frac{1}{2 \bar{a}_{01}}\left[w_{01}(x)-\hat{w}_{01}(x)\right]
$$

and, therefore, from (54), that:

$$
\bar{a}_{01} c_{00}(k)=\frac{1}{2} \int_{-\infty}^{0} d x\left[w_{01}(x)-\hat{w}_{01}(x)\right] \exp (i k x) .
$$

Introducing this expression, and the definition (19) of $c_{01}$ into (61), we find that:

$$
C(k)=\frac{1-\frac{1}{2} i k \int_{-\infty}^{0} d x\left[w_{01}(x)-\hat{w}_{01}(x)\right] \exp (i k x)}{1-i k \int_{-\infty}^{0} d x w_{01}(x) \exp (i k x)}
$$

For the simple kernel (3), it is easily shown that $\phi_{01}(x)=(1-x / x)^{1 / 2}=-\hat{\phi}_{01}(1-x)$, and thus, that $w_{01}(x)=1-(x-1 / x)^{1 / 2}$. The integrals appearing in (70) are, then, readily identified as Hankel functions of the second kind, with the result that:

$$
C(k)=\frac{H_{1}^{(2)}(k / 2)}{H_{1}^{(2)}(k / 2)+i H_{0}^{(2)}(k / 2)} .
$$

This is the so-called "Theodorsen function" (cf. [1] or [3]) which plays a key role in unsteady aerodynamics and aeroelasticity.

It is clear, then, that (70) constitutes a generalized Theodorsen function for kernels of the form (2). The exact behavior of $C(k)$ is determined by the particular steady kernel, $K_{0}$. We note, however, that $C(0)=1$ for all $K_{0}$. If $K_{0}$ decays sufficiently rapidly at infinity (e.g. exponentially) then $C(k)$ may be expanded in a Taylor series for low frequencies. If $K_{0}$ decays only algebraically (as does (3)) then $C(k)$ will generally have a branch point at $k=$ 0 . At high frequencies, on the other hand, we expect that the terms involving $w_{01}(x)$ in $(70)$ will dominate both the numerator and denominator (since, in general, $w_{01}(x) \gg \hat{w}_{01}(x) \rightarrow$ 1 as $x \rightarrow 0$ ). Thus we find that $C(k) \rightarrow 1 / 2$ as $k \rightarrow \infty$. The high- and low-frequency limits of $C(k)$, then, are common to all kernels of the form (2) (in fact it was in order that this property be satisfied that the constant $\bar{a}_{01}$, rather than any other, was introduced into the definition (61)).

As a practical matter it may, in some cases, be more convenient to compute $C(k)$ from the equivalent formula:

$$
\begin{aligned}
C(k)= & \frac{1}{2} \frac{\int_{0}^{1} K(t-x ; k)\left[\phi_{01}(t)+\hat{\phi}_{01}(t)\right] d t}{\int_{0}^{1} K(t-x ; k) \phi_{01}(t) d t} \\
& \frac{1+\frac{1}{2} \int_{0}^{1} d t K_{1}(t-x ; k)\left[\phi_{01}(t)+\hat{\phi}_{01}(t)\right]}{1+\int_{0}^{1} d t K_{1}(t-x ; k) \phi_{01}(t)}
\end{aligned}
$$


where

$$
K_{1}(x ; k)=K(x ; k)-K_{0}(x)
$$

is the "unsteady part" of the kernel $K$. Eq. (72), which is valid for any $x$ in $(0,1)$, can be obtained most easily from (65).

If the functions $w_{01}$ and $\hat{w}_{01}$ can be found in reasonably simple analytic form, then $C(k)$ may most easily be computed from their half Fourier transforms via (70). Alternatively, if these two functions can be approximated in terms of simple functions (over the domain $(-\infty, 0))$ then $(70)$ may, again, be more convenient than (72).

Any purely numerical evaluation of $C(k)$, however, will be more easily accomplished by performing the quadratures in (72), which are over a finite interval. Moreover, we note that the functions $\phi_{01}, \hat{\phi}_{01}$ which appear in (72) must be evaluated anyway to construct the resolvent, whereas $w_{01}$ and $\hat{w}_{01}$, which appear in (70), do not appear anywhere else in the solution.

8. Applications. Integral equations of type (1) occur in the general theory of thin airfoils in parallel flow fields (this theory is developed in [5]). In such problems $\phi(x)$ is the lifting pressure on the airfoil and $w(x)$ is an effective downwash or vertical velocity associated with the airfoil motion. The parameter $k$ is the ("reduced") frequency of that motion in units of the transit time of a reference (e.g. free stream) particle crossing the chord. The kernel function $K$ is determined by the undisturbed flow profile and the frequency of the airfoil motion. In general the frequency dependence of $K$ is much more complicated than that assumed in Eq. (2). However, in certain circumstances, an equation of the form given by (2) may serve as a useful approximation.

Kernel functions which do satisfy conditions (2) exactly arise for incompressible stratified flows (if gravity is neglected) in which the density gradient is normal to the plane of the airfoil. The steady kernel $K_{0}$ is real and anti-symmetric for such flows. It is determined by the density profile from the following set of relations:

$$
K_{0}(x)=\frac{1}{2 \pi} \int_{-\infty}^{\infty} K_{0}^{*}(\alpha) \exp (i \alpha x) d \alpha,
$$

where

$$
\begin{aligned}
& K_{0}^{*}(\alpha)=K_{0^{+}}{ }^{*}(\alpha) K_{0-}{ }^{*}(\alpha) /\left(K_{0-}{ }^{*}(\alpha)-K_{0+}{ }^{*}(\alpha)\right), \\
& K_{0^{ \pm}}{ }^{*}(\alpha)=i \frac{|\alpha|}{\alpha} V(z= \pm 0 ; \alpha), \\
& \frac{1}{|\alpha|} \frac{d V}{d z}=\frac{1}{\rho(z)}-\rho(z) V^{2} ; \rho V \rightarrow \pm 1 \quad \text { as } \quad z \rightarrow \mp \infty
\end{aligned}
$$

Here $z$ is the normal distance from the plane of the airfoil measured in units of chord length and $\rho(z)$ is the prescribed density profile of the undisturbed fluid. (The Riccatti equation (77) is equivalent to a linear second-order equation describing the pressure variations above and below the airfoil). The boundary conditions imposed on (77) ensure the decay of disturbances in the far field. If the flow is bounded by a rigid or free surface at $z=D$ (say) the appropriate boundary condition there is $V=0$ or $1 / V=0$, corresponding to zero vertical velocity on a rigid wall or zero pressure on a free surface (these cases can be viewed as special density distributions in the infinite domain, i.e. $\rho=\infty$ or $\rho=0$ for $z>$ $D)$. It will be noted that various potential flow problems of interest fall within this 
category, including wind tunnel wall and ground effects [7] and hydrofoil free surface interactions [8].

The restriction to gravity-free flows is part of a wider restriction to media which cannot support waves. To see this we observe that Eq. (2) is equivalent to the relation:

$$
K^{*}(\alpha ; k)=\frac{\alpha}{\alpha-k} K_{0}^{*}(\alpha)
$$

between the Fourier transforms (defined as in (74)). Eq. (78) implies that $k / \alpha=1$ is the only characteristic speed of the free system (since $1 / K^{*}(\alpha ; k)=0$ is the full dispersion relation, whether (78) holds or not). Consequently, (78) cannot hold for any wavesupporting media, e.g. stratified flows in a gravity field or compressible flows.

Having made this limitation clear, we can now remove it. For, although we have presumed throughout the development of Secs. (1-7) that $K_{0}$ is the steady kernel (i.e. independent of $k$ ), at no time did we use this fact. Thus, the results obtained are valid for any two kernels related by (2) (or (78)) whether or not $K_{0}$ is independent of $k$. In the general case the "solution" obtained here amounts simply to a transformation from one unsteady problem to another. The utility of this transformation must be judged on the basis of whether or not the transformed problem (for $\phi_{00}$ and $\phi_{01}$ ) is simpler than the original problem (for $h$ and $\phi_{1}$ ). This will be the case, for instance, if $K_{0}$ is antisymmetric (or more generally, if $K_{0}{ }^{*}(\alpha)$ is anti-symmetric about some point $\alpha=\alpha_{0}$ ), or if there is a reduction in the number of parameters involved. As an example we may note that the problem for subsonic potential flow over an oscillating airfoil can be reduced, using the methods of this paper, from its original two-parameter asymmetric form to a oneparameter antisymmetric form. As a result the resolvent of that problem (heretofore available only in terms of infinite series of Mathieu functions [9]) can be expressed in terms of a single one-parameter function of $x$.

11. Conclusions. It has been shown that Theodorsen's solution for the pressure distribution on an oscillating airfoil in unbounded incompressible flow is a special application of a general transformation applicable to any singular integral equation of the airfoil type. For some problems, such as that of an airfoil oscillating in an incompressible stratified flow or a bounded potential flow, this transformation provides a complete separation of the spatial and frequency dependence of the solution. In other problems the transformation may be of value in reducing the number of parameters in the problem or the number of "primitive" functions required to describe the solution.

In those cases where a complete separation of the frequency dependence is possible, the response of the system to an arbitrary disturbance can be obtained by Fourier superposition. The resulting temporal response $\phi(x, t)$ contains three contributions: one from the instantaneous forcing term $w(x, t)$, one from the instantaneous rate of change $(\partial / \partial t) w(x, t)$, and one from the cumulative effect of the preceding motion which appears in the form of a Duhamel superposition integral.

\section{REFERENCES}

[1] T. Theodorsen, General theory of aerodynamic instability and the mechanism of flutter, NACA Report 496, 1935

[2] H. G Küssner, Zusammenfassender Berich über den instationaeren Auftrieb von Fluegeln, Luftfahrtforschg. 13., 12, December 1936

[3] R. L. Bisplinghoff, H. Ashley, and R. L. Halfman, Aeroelasticity, Addison-Wesley, 1955 
[4] M. H. Williams, The resolvent of singular integral equations, Quart. App. Math. 35, 99-110 (1977)

[5] M. H. Williams, General theory of thin wings in inviscid parallel shear flows, Princeton University AMS Report 1269, March 1976

[6] High speed aerodynamics and jet propulsion, Vol. VI: General theory of high speed aerodynamics, W. R. Sears, ed., Princeton University Press, 1964

[7] W. P. Jones, Wind-tunnel wall interference effects on oscillating aerofoils in subsonic flow, British ARC R \& M 2943, 1953

[8] S. E. Widnall, Unsteady loads on hydrofoils including free surface effects and cavitation, MIT Fluid Dyn. Res. Lab., Report 64-2, June 1964

[9] D. E. Williams, On the integral equations of two dimensional subsonic flutter derivative theory, British ARC. R \& M. 3057, 1958

[10] M. Erdélyi et al., Higher transcendental functions, Vol. 1, McGraw-Hill, 1953 